\title{
Pancreas allograft biopsies procedure in the management of pancreas transplant recipients
}

\author{
Jiao Wan, Jiali Fang, Guanghui Li, Lu Xu, Wei Yin, Yunyi Xiong, Luhao Liu, Tao Zhang, Jialin Wu, \\ Yuhe Guo, Junjie Ma, Zheng Chen
}

Organ Transplant Center, Second Affiliated Hospital of Guangzhou Medical University, Guangzhou 511447, China

Contributions: (I) Conception and design: J Wan; (II) Administrative support: J Ma, Z Chen; (III) Provision of study materials or patients: J Fang, G Li, L Xu, W Yin, Y Xiong, L Liu, T Zhang, J Wan; (IV) Collection and assembly of data: L Wu, Y Guo, J Wan; (V) Data analysis and interpretation: J Wan; (VI) Manuscript writing: All authors; (VII) Final approval of manuscript: All authors

Correspondence to: Zheng Chen. Organ Transplant Center, Second Affiliated Hospital of Guangzhou Medical University, Guangzhou 511447, China.

Email: docchenzheng@163.com.

\begin{abstract}
Pancreas transplantation is an effective therapy for diabetic patients, which can significantly improve the survival rate and quality of life of diabetic patients. According to the international registration of pancreas transplantation center, the global total pancreas transplantation has reached more than 80,000 cases by 2017 , including pure pancreas transplantation and simultaneous pancreas-kidney transplantation (SPK). With the development and application of a new type of immunosuppressant, with the gradual maturity of organ preservation technology and surgical technology, the pancreas transplantation has rapidly on a global scale. However, pancreas transplantation still has more problems than limit its development compared with other organ transplantation. For example, the early diagnosis and treatment of pancreatic rejection are of considerable significance to the prognosis of pancreas transplantation. Some surveillance methods of diagnosis have been used increasingly, among which the histopathological diagnosis is particularly important. The first Banff schema for the histological diagnosis of pancreas rejection has been published, which primarily dealt with the diagnosis of acute T-cell-mediated rejection (ACMR). In recent years, antibodymediated rejection (AMR) has been more emphasized as the primary cause of graft failure. The Banff pancreas allograft rejection grading schema was updated in 2011 by a broad-based multidisciplinary panel, presenting comprehensive guidelines for the diagnosis of AMR.
\end{abstract}

Keywords: Pancreas transplantation, pancreas allograft rejection; acute T-cell-mediated rejection (ACMR); antibody-mediated rejection (AMR); Banff schema; histopathological diagnosis; biopsy technique, ultrasonic guidance

Submitted Sep 20, 2019. Accepted for publication Nov 05, 2019.

doi: 10.21037 /gs.2019.12.01

View this article at: http://dx.doi.org/10.21037/gs.2019.12.01

\section{Introduction}

Pancreas transplantation is a practical treatment choice for patients with diabetes mellitus (DM) (1). The operation of pancreas transplantation divides into three diverse ways depending on the patient's native renal function, simultaneous pancreas-kidney transplantation (SPK), pancreas after kidney transplantation (PKA) and the pancreas transplantation alone (PTA) (2). SPK is the most common type of pancreas transplantation performed in patients with end-stage renal disease (ESRD). The slower progression of pancreas transplantation in comparison to other allograft transplants was attributed to surgery technical and short-/long-term outcomes. Since the late 1980s, the results of pancreas transplantation have continued to improve, with current 1 -year survival rate of patients after pancreas transplantation exceeding $95 \%$, and 3-year survival rate exceeding $90 \%$. The 1 - and 3 -year 
graft survival rates are $83 \%$ and $77 \%$, respectively (3). The improvement of transplantation results from the increase of transplantation technology, new and more specific immunosuppressive drugs, anti-infection therapy and correct diagnosis and prompt treatment of rejection. Unfortunately, despite a visible reduction in the rates of early acute rejection, long-term outcomes have not improved markedly, on account of intractable rejection or inexorable progression to graft fibrosis which emphasize the significance of early correct diagnosis (4-6).

The diagnosis of transplanted pancreas rejection combines clinical diagnosis with histological diagnosis. The first Banff schema for histological diagnosis of pancreas rejection has been published in 2008 and updated in 2011 by a broad-based multidisciplinary panel $(7,8)$.

\section{Clinical diagnosis}

Most of the patients with pancreas transplantation have no or mild symptoms, such as graft slight tenderness, intestinal paralysis, and acute abdominal pain, but it is relatively rare and tough to identify with pancreatitis, due to not specific symptoms, so a separate clinical symptoms do not have the significance of diagnosis of transplanted pancreas rejection. The clinical diagnosis relies heavily on laboratory markers of acinar cell injury.

Pancreatic rejection markers can be divided into two parts: exocrine rejection markers and endocrine rejection markers. Rejection markers were determined by serologic test (all drainage methods) or urine test (bladder drainage technique only) based on the surgical treatment of the pancreatic exocrine department. Urinary amylase has been widely used as rejection marker for pancreatic transplantation with bladder drainage. For pancreatic transplantation with intestinal drainage, a combination of exocrine markers (e.g., amylase, lipase, positive trypsin) and endocrine markers (e.g., glucose metabolic rate) can be used. However, all current rejection markers are not 100\% specificity or sensitivity for the diagnosis of transplanted pancreatic rejection. A majority of pancreas rejection cases have detected the increase in serum amylase or lipase, with biopsy-proven rejection approximately $80 \%(9,10)$. Also, the reduction of amylase in patients with bladder exocrine drainage is proved to accounting for $53 \%$ of cases diagnosed histologically $(9,11)$.

For SPK recipients, renal function can be detected by serum creatinine level, and the rejection of pancreas can be reflected some extent. However, the absolute diagnostic value of this method is questionable because the rejection of only one organ in patients is not rare. In a large sample study of SPK recipients, when both kidney and pancreas biopsies were performed, $65 \%$ occurred graft rejection both, $22 \%$ involved the pancreas alone, and $13 \%$ involved the kidneys alone $(12,13)$.

\section{Histological diagnosis}

Clinical symptoms and laboratory markers are neither lack of specificity, the transplantation of pancreas, as with all other organs, transplantation biopsy is the gold standard for the diagnosis of rejection.

\section{The method of transplanted pancreas biopsy}

Fine needle aspiration biopsy is a useful skill for diagnosis of pancreatic rejection in early stage, which can detect the components of cell and humoral rejection by aspiration of infiltrated mononuclear cells. The most significant advantage of this approach is that it is usually performed under the guidance of ultrasound with minimum damage and risk. The main complications are bleeding, infection, or pancreatic fistula, etc., but the incidence rate is low. The disadvantage is that there is a failure rate of approximately $30 \%$, especially in the presence of peripancreatic effusion or graft thrombosis, and the obtained sample can only be used once, and the diagnosis is hugely dependent on the experience of pathologists to a large extent.

Fine needle puncture biopsy is a more widely used biopsy method for pancreas transplantation recently. Puncture biopsy also has potential complications, including intraperitoneal bleeding, pancreatitis, and pancreatic leakage, so it is more cautious than renal biopsy.

Percutaneous biopsy of the transplanted pancreas is mainly conducted under the guidance of ultrasound alone, computer tomographic alone or combined ultrasound with computed tomography, which can significantly reduce the risk of puncture on the premise of obtaining enough biopsy tissue (13). Vital signs should be carefully checked six to eight hours after biopsy. Percutaneous biopsies are low cost and short hospital stay and can be performed in out-patient. The tissue recovery rate of percutaneous biopsy is $87 \%$ to $92 \%$, and the needle is usually 18 - or 20 -gauge needle (14). A report from the University of Maryland suggests that to avoid hemorrhagic complications, the pancreas should be located under ultrasound guidance, and the main blood vessels should be distinguished, and then puncture points 
that can prevent the large blood vessels and overlap the intestine should be selected. In a study of 63 recipients who underwent computer tomographic guided biopsy, 57 patients obtained adequate tissue samples and $8 \%$ patients developed mild complications, including transient hyperamylasemia (relieved within 2 to 4 days) and transient mild hematuria, $3 \%$ had major complications, one patient needed surgical exploration and only one case occurred bleeding, but stopped immediately after using No. 18 embolic gelatin through the embolization biopsy pathway (14).

For pancreatic transplant recipients with bladder drainage, cystoscope biopsy through duodenum is the best way to obtain adequate samples with a low incidence of complications. The rate of massive hematuria is less than $10 \%$, and even though it occurs, continuous bladder irrigation with a three-cavity catheter can be an effective treatment. Also, biopsy-associated pancreatitis, in which serum amylase levels are elevated after biopsy, is uncommon and self-healing. With the development of percutaneous biopsy technology, the more invasive cystoscope biopsy has become less adopted and more expensive.

The laparoscopic biopsy is suitable for the recipient of intestinal drainage transplantation, and percutaneous biopsy is not safe (mainly because the graft is covered by the loop of the small intestine). The technical problem is that patients with pancreatic transplants often develop abdominal adhesions, even with high doses of hormones. Compared with percutaneous biopsy, the advantage is that the bleeding can be stopped easily by electrocoagulation under the endoscope. Recently a laparoscopic biopsy of transplanted pancreas research shows that $91.2 \%$ of the laparoscopic biopsy (145 in 159 cases received enough pancreatic tissue), can obtain adequate samples and make a diagnosis. They were not able to perform the pancreas biopsy laparoscopically in 8 patients because of extensive adhesions (in 6 cases), the presence of an en-bloc pediatric kidney-pancreas transplant that did not allow them to reach the pancreas (in 1 case), and in the other case the pancreas biopsy was not done because of a laceration of the graft duodenum $(15,16)$.

When adequate diagnostic specimens cannot be obtained by the above methods, open biopsy can be selected, which has a high incidence of complications, even graft loss, and low cost/benefit ratio.

Horneland R' study support that, to better vision and obtain the pancreas sample, it performs duodenal anastomosis instead of duodenal jejunostomy, the results show that the endoscopic pancreatic biopsy can retrieve a more representative tissue sample. Although duodenal anastomosis has a higher rate of thrombosis $(23 \% v s$. $5 \%$ ) and the second surgery ( $48 \%$ vs. $30 \%$ ), and lower graft survival ( $80 \%$ vs. $88 \%$ ) compared with duodenal jejunostomy, but these problems can be solved slowly as the development technical (17).

Laftavi firstly puts forward the rules of the transplanted pancreas biopsy. According to the rules, no matter what kind of drainage technology, the first choice for hospitalized patients is the percutaneous biopsy. If failure, according to the unusual ways of exocrine drainage, takes the following options: bladder drainage of the pancreas in recipients, cystoscope biopsy should, if they fail, any laparoscopic biopsy; In intestinal drainage recipients, a laparoscopic biopsy was selected. If all the above techniques fail and exact histopathological diagnosis is necessary, the final approach in all recipients is an open biopsy.

Biopsy site: one study reported that the pancreatic tail was better than the pancreatic head, but the number of studies was small.

To qualify puncture biopsy specimens, it is recommended that there be at least three lobules and corresponding interlobular septa with venous and ductal branches of the pancreas. Due to the difficulty of arterial sampling, which is especially crucial for diagnosis, it is recommended to note in the pathological report if there is no artery in the specimen.

\section{Pancreatic biopsy specimen processing}

After fixed with conventional neutral formalin fixative, the tissue is dehydrated, embedded, and sectioned in turn. To make more accurate diagnosis, it is recommended to cut more than or equal to 10 continuous or interphase sections for different staining. (I) 3 discontinuous sections for $\mathrm{HE}$ staining; (II) 1 for Masson three staining; (III) 1 section was used for C4d immunohistochemical staining; (IV) the remaining sections are used for other examinations. The other sections include cytomegalovirus (CMV) staining, PAS staining to look at the acini structure. Also, in patients who have biopsies due to hyperglycemia, insulin, and glucagon staining must be performed to demonstrate selective loss of islet $\mathrm{B}$ cells, which suggest a recurrence of autoimmune disease.

Programmed biopsies are performed at a point in time, regardless of the function of the transplanted organ. There are few reports about procedural biopsy up to now. Some reports suggest that routine biopsy at 1, 3, 6, and 12 months after surgery is safe and effective, and can 
detect graft rejection early. Instead, a retrospective study on evaluation of Maryland II level (slight) rejection found procedural biopsy results after line, Maryland II grade rejection rarely progress to more severe inflammation. Also, procedural biopsy will inevitably increase the incidence of complications, and the biopsy cost is high. Therefore, most transplant centers only conduct graft biopsy on patients with indications, which are determined according to the changes in laboratory parameters or clinical symptoms.

\section{Pathological diagnosis}

According to the 2007 Banff pancreatic allograft rejection classification scheme, the diagnosis was made by 2 experienced pathologists. Histopathological changes of the transplanted pancreas were classified into 6 diagnostic categories: (I) normal; (II) uncertainty; (III) cell-mediated rejection types: acute (grade levels I, II, III) and chronic activity; (IV) antibody-mediated rejection (AMR): hyperacute, acute and chronic activity; (V) of chronic allograft rejection/graft sclerosis (I stage, II stage, III period); (VI) other histological diagnosis.

For transplantation of pancreas biopsy specimens, cellmediated acute homograft rejection and chronic rejection/ graft hardening is one of the most common diagnosis, can be divided into different levels, in the future with the development of the transplanted pancreas pathology diagnosis, classification scheme would join score way, with the histologic findings of lesions more accurate evaluation.

\section{Discussion}

For whole-organ pancreas transplants, the pancreas is usually anastomosed to the recipient's intestine or bladder through a segment of the duodenum. For patients with bladder drainage, duodenal puncture biopsy can be performed through the cystoscope. For patients with intestinal drainage, duodenal specimens can be obtained by endoscopic ultrasonography (18). The standardized sampling site is to take the mucosal area, generally do not need to take the submucosal area. One to two pieces of tissue were sampled from each site and quickly placed into the medium formalin, paraffin sections were made, and HE staining was performed for initial evaluation, as well as some special staining, immunohistochemical examination and in situ hybridization.

Due to the slower progress of pancreas transplant in China, the pancreas biopsy is performed barely in transplant centers. It is urgently necessary to produce a systematic and rational approach of transplant pancreas biopsy which can guide clinical treatment and judge the prognosis. The first, a proper pancreas biopsy method will be favorable to maximize diagnostic yield and make precise histopathological diagnosis, encourage the use of pancreas graft biopsies and should result in improved pancreas transplantation outcomes.

\section{Acknowledgments}

None.

\section{Footnote}

Conflicts of Interest: The authors have no conflicts of interest to declare.

Ethical Statement: The authors are accountable for all aspects of the work in ensuring that questions related to the accuracy or integrity of any part of the work are appropriately investigated and resolved.

\section{References}

1. Larsen JL, Colling CW, Ratanasuwan T, et al. Pancreas transplantation improves vascular disease in patients with type 1 diabetes. Diabetes Care 2004;27:1706-11.

2. Becker BN, Becker YT, Odorico JS, et al. Simultaneous pancreas-kidney and pancreas transplantation. Minerva Urol Nefrol 2002;54:213-26.

3. Andreoni KA, Brayman KL, Guidinger MK, et al. Kidney and pancreas transplantation in the United States 19962005. Am J Transplant 2007;7:1359-75.

4. Allen RD, Grierson JM, Ekberg H, et al. Longitudinal histopathologic assessment of rejection after bladderdrained canine pancreas allograft transplantation. Am J Pathol 1991;138:303-12.

5. Humar A, Khwaja K, Ramcharan T, et al. Chronic rejection: The next major challenge for pancreas transplant recipients. Transplantation 2003;76:918-23

6. Stratta RJ. Patterns of graft loss following simultaneous kidney pancreas transplantation. Transplant Proc 1998;30:288.

7. Drachenberg CB, Odorico J, Demetris AJ, et al. Banff schema for grading pancreas allograft rejection: working proposal by a multi-disciplinary international consensus panel. Am J Transplant 2008;8:1237-49. 
8. Drachenberg CB, Torrealba JR, Nankivell BJ, et al. Guidelines for the diagnosis of antibody-mediated rejection in pancreas allografts-updated Banff grading schema. Am J Transplant 2011;11:1792-802.

9. Benedetti E, Najarian JR, Gruessner AC, et al. Correlation between cystoscopic biopsy results and hypoamylasuria in bladder-drained pancreas transplants. Surgery 1995;118:864-72.

10. Klassen DK, Hoen-Saric EW, Weir MR, et al. Isolated pancreas rejection in combined kidney pancreas transplantation. Transplantation 1996;61:974-77.

11. Nankivell BJ, Allen RD, Bell B, et al. Factors affecting urinary amylase excretion after pancreas transplantation. Transplant Proc 1990;22:2156-7.

12. Gaber AO, Gaber LW, Shokouh-Amiri MH, Et al. Percutaneous biopsy of pancreas transplants. Transplantation 1992;54:548-50.

13. Kuo PC, Johnson LB, Schweitzer EJ, et al. Solitary pancreas allografts: the role of percutaneous biopsy and standardized histologic grading of rejection. Arch Surg

Cite this article as: Wan J, Fang J, Li G, Xu L, Yin W, Xiong Y, Liu L, Zhang T, Wu J, Guo Y, Ma J, Chen Z. Pancreas allograft biopsies procedure in the management of pancreas transplant recipients. Gland Surg 2019;8(6):794-798. doi: 10.21037/ gs.2019.12.01
1997;132:52-7.

14. Munn SR, Engen DE, Barr D, et al. Differential diagnosis of hypoamylasuria in pancreas allograft recipients with urinary exocrine drainage. Transplantation 1990;49:359-62.

15. Klassen DK, Weir MR, Cangro CB, et al. Pancreas allograft biopsy: Safety of percutaneous biopsy-results of a large experience. Transplantation 2002;73:553-5.

16. Uva PD, Odorico JS, Giunippero A, et al. Laparoscopic Biopsies in Pancreas Transplantation. Am J Transplant 2017;17:2173-7.

17. Horneland R, Paulsen V, Lindahl JP, et al. Pancreas transplantation with enteroanastomosis to native duodenum poses technical challenges--but offers improved endoscopic access for scheduled biopsies and therapeutic interventions. Am J Transplant 2015;15:242-50.

18. Nordheim, E, Horneland R, Aandahl EM, et al. Pancreas transplant rejection episodes are not revealed by biopsies of the donor duodenum in a prospective study with paired biopsies. Am J Transplant 2018;18:1256-61. 